\title{
72. EVOLUTION OF THE ORBITS OF SELECTED MINOR PLANETS DURING AN INTERVAL OF 1000 YEARS
}

\author{
M. A. DIRIKIS \\ Astronomical Observatory, Latvian State University, Riga, U.S.S.R.
}

\begin{abstract}
The long-term evolution of the orbits of 944 Hidalgo, 1036 Ganymed and 1134 Kepler is investigated.
\end{abstract}

The investigations of the evolution of cometary and asteroidal orbits performed by Kazimirchak-Polonskaya $(1967,1968)$, Belyaev and Chebotarev (1968), and Chebotarev et al. (1970) are very precise, but they are limited to a $400-\mathrm{yr}$ time interval (1660-2060), because precise coordinates of the outer planets are available only for this interval (Eckert et al., 1951).

For our studies we have made use of approximate coordinates of the major planets computed analytically using the principal perturbation terms. It thus becomes possible, in theory, to extend the studies of orbital evolution over unlimited time intervals. One must pay attention to two points, however, which restrict the possibility of obtaining plausible results:

(1) The accumulation of error after a large number (10000 or more) of integration steps.

(2) The approximate theory used for the calculation of the coordinates of perturbing bodies is not sufficiently accurate if the time interval elapsed from the initial osculation epoch is very long (1000 yr or more).

Consequently we have generally limited our calculations to a time interval of about $1000 \mathrm{yr}$. We make use of a variation-of-elements method, since the changes in the elements are relatively small and we can use larger integration steps. Perturbations by the planets Venus to Saturn are taken into account.

So far we have studied the evolution of the orbits of the following asteroids: 944 Hidalgo, 1036 Ganymed, and 1134 Kepler. The orbits of these asteroids all have high eccentricities and are similar to the orbits of comets.

One of the most interesting of all asteroids, 944 Hidalgo has not only one of the highest orbital eccentricities (being exceeded in this respect only by 1566 Icarus), but also one of the highest inclinations (exceeded only by 1580 Betulia) and the greatest semimajor axis. Its evolution has therefore been investigated over an interval of more than $2000 \mathrm{yr}$ (from 670 to 2900 ) (see Table I). This minor planet is exceptional in that it can come closer to Jupiter than the other planets can (see Table II). The closest encounters of 944 with Jupiter are as follows: $0.43 \mathrm{AU}$ in 1211, 0.36 in 1673 and 0.50 in 2823. Marsden (1970) has found that all the minor planets avoid encounters with Jupiter except for 944 Hidalgo. The two planets having the next closest encounters are 279 Thule and 334 Chicago, which can approach to a distance of 1.1 AU from Jupiter. According to Marsden, 944 Hidalgo is a comet. 
TABLE I

Elements of 944 Hidalgo

\begin{tabular}{rllllll}
\hline Yr & $\omega$ & $\Omega$ & $i$ & $\varphi$ & $\mu$ & $a(\mathrm{AU})$ \\
\hline 701 & 71.9 & 32.3 & 48.8 & 33.8 & $266 " 89$ & 5.6120 \\
903 & 69.5 & 30.6 & 47.9 & 34.8 & 260.76 & 5.6996 \\
1104 & 64.4 & 29.0 & 47.9 & 35.9 & 272.72 & 5.5317 \\
1300 & 61.6 & 27.0 & 46.6 & 37.1 & 265.68 & 5.6291 \\
1510 & 59.9 & 25.3 & 45.4 & 38.6 & 266.83 & 5.6129 \\
1713 & 59.3 & 23.3 & 43.3 & 40.0 & 254.30 & 5.7957 \\
1907 & 56.8 & 21.8 & 43.0 & 40.6 & 255.91 & 5.7714 \\
1964 & 57.6 & 21.0 & 42.5 & 41.0 & 252.75 & 5.8193 \\
2101 & 55.8 & 20.1 & 42.4 & 41.6 & 260.17 & 5.7082 \\
2309 & 55.1 & 18.1 & 41.6 & 42.2 & 253.27 & 5.8115 \\
2502 & 54.1 & 16.4 & 41.1 & 42.9 & 258.19 & 5.7374 \\
2709 & 53.4 & 14.2 & 40.5 & 43.5 & 258.21 & 5.7371 \\
2903 & 51.4 & 12.2 & 39.2 & 44.6 & 258.68 & 5.7301 \\
\hline
\end{tabular}

TABLE II

Minimal distances (AU)

\begin{tabular}{llll}
\hline Asteroid & Earth & Mars & Jupiter \\
\hline 944 Hidalgo & 1.19 & 0.85 & 0.36 \\
1036 Ganymed & 0.33 & 0.07 & 1.90 \\
1134 Kepler & 0.43 & 0.08 & 1.52 \\
\hline
\end{tabular}

The changes in the orbital elements of asteroids 1036 and 1134 are shown in Tables III and IV. In spite of the absence of close encounters with Jupiter we see that these changes have the same range as in the case of 944 Hidalgo.

TABLE III

Elements of 1036 Ganymed

\begin{tabular}{lllllll}
\hline Yr & $\omega$ & $\Omega$ & $i$ & $\varphi$ & $\mu$ & $a(\mathrm{AU})$ \\
\hline 1351 & 115.8 & $232 \circ 8$ & 22.8 & 35.0 & 816.62 & 2.6627 \\
1451 & 118.3 & 229.8 & 23.3 & 34.6 & 815.64 & 2.6648 \\
1551 & 121.0 & 227.0 & 23.8 & 34.3 & 816.05 & 2.6640 \\
1651 & 124.4 & 223.5 & 24.5 & 33.9 & 816.26 & 2.6635 \\
1750 & 126.7 & 221.0 & 25.1 & 33.4 & 815.85 & 2.6644 \\
1850 & 128.7 & 218.7 & 25.6 & 33.1 & 816.43 & 2.6631 \\
1950 & 131.1 & 216.3 & 26.3 & 32.9 & 818.60 & 2.6584 \\
2050 & 133.4 & 214.0 & 27.0 & 32.2 & 817.26 & 2.6613 \\
2150 & 135.1 & 212.1 & 27.5 & 31.5 & 815.44 & 2.6653 \\
2250 & 136.9 & 210.3 & 28.1 & 31.1 & 815.88 & 2.6643 \\
2350 & 139.4 & 208.2 & 28.8 & 30.6 & 817.36 & 2.6611 \\
2450 & 141.0 & 206.6 & 29.3 & 30.0 & 816.38 & 2.6632
\end{tabular}


TABLE IV

Elements of 1134 Kepler

\begin{tabular}{llrllll}
\hline $\mathrm{Yr}$ & $\omega$ & $\Omega$ & \multicolumn{1}{l}{$i$} & $\varphi$ & $\mu$ & $a(\mathrm{AU})$ \\
\hline 1349 & $305 \circ 7$ & 23.8 & 12.8 & 28.9 & 807.92 & 2.6818 \\
1450 & 310.7 & 20.1 & 13.2 & 28.6 & 806.01 & 2.6860 \\
1551 & 315.4 & 16.6 & 13.6 & 28.8 & 809.59 & 2.6781 \\
1648 & 319.1 & 14.0 & 14.0 & 28.3 & 806.89 & 2.6841 \\
1749 & 323.6 & 11.1 & 14.4 & 28.3 & 808.19 & 2.6812 \\
1850 & 326.3 & 9.0 & 14.6 & 28.0 & 807.23 & 2.6833 \\
1951 & 330.5 & 6.7 & 15.0 & 27.8 & 806.87 & 2.6841 \\
2048 & 333.1 & 4.9 & 15.3 & 27.6 & 806.55 & 2.6848 \\
2149 & 336.9 & 2.8 & 15.6 & 27.5 & 806.34 & 2.6853 \\
2250 & 339.5 & 1.3 & 15.8 & 27.3 & 806.19 & 2.6856 \\
2351 & 343.0 & 359.5 & 16.0 & 27.2 & 805.44 & 2.6873 \\
2448 & 345.3 & 358.0 & 16.2 & 27.3 & 807.96 & 2.6817 \\
\hline
\end{tabular}

In conclusion we can state that there is a significant difference between the motions of comets and asteroids:

(1) Close encounters with the major planets occur less frequently for asteroids than for comets.

(2) These encounters are never as close.

Consequently, the changes in the orbital elements of asteroids are never catastrophic. Small periodic (e.g., in $a$ ) and slow secular (e.g., in $\omega$ and $\Omega$ ) variations prevail in the case of asteroids. The encounters cause the evolutionary changes in the elements to be accelerated, but in general this influence is of short duration.

\section{References}

Belyaev, N. A. and Chebotarev, G. A.: Astron. Tsirk. No. 480.

Chebotarev, G. A., Belyaev, N. A., and Eremenko, R. P.: 1970, Byull. Inst. Teor. Astron. 12, 82.

Eckert, W. J., Brouwer, D., and Clemence, G. M.: 1951, Astron. Pap. Washington 12.

Kazimirchak-Polonskaya, E. I.: 1967, Trudy Inst. Teor. Astron. 12, 3.

Kazimirchak-Polonskaya, E. I.: 1968, Astronomie 82, 323.

Marsden, B. G.: 1970, Astron. J. 75, 206. 